\title{
Is Conversion from Mycophenolate Mofetil to Enteric-Coated Mycophenolate Sodium Justifiable for Gastrointestinal Quality of Life?
}

\author{
Kyle M. Gardiner ${ }^{1,2} \cdot$ Susan E. Tett $^{1} \cdot$ Christine E. Staatz $^{1}$
}

Published online: 13 November 2018

(c) The Author(s) 2018

\begin{abstract}
Background Globally, enteric-coated mycophenolate sodium (EC-MPS) is replacing mycophenolate mofetil (MMF) in maintenance immunosuppressant regimens. The predominant reason for conversion is the purported improvement in gastrointestinal (GI) quality of life. This paper considers the level of bias associated with studies comparing EC-MPS and MMF for GI-related improvement and provides insight into whether conversion is supported by evidence.

Methods Using a pre-determined protocol, a literature search was conducted. Full-text review, data extraction and risk of bias analysis was conducted by two independent authors using the Cochrane domain-based evaluation of risk of bias. The review was reported according to the preferred reporting items for systematic reviews and meta-analyses.

Results Twenty-nine studies were included in risk of bias analysis. Of these, only three were deemed a low risk of bias. Across these three studies, there were no statistically significant differences in the proportion of GI-related adverse events nor was there a significant difference in the GI-related quality of life between EC-MPS- and MMF-treated patients in these data. Conclusion There was a high risk of bias across the 29 studies investigating conversion from MMF to EC-MPS for potential improvement in GI-related quality of life. The consolidated results of the three studies with low risk of bias suggest no evidence to convert patients stabilised on MMF. If a patient experiences GI-related adverse events whilst taking MMF, other methods should be explored before conversion to EC-MPS.
\end{abstract}

Electronic supplementary material The online version of this article (https://doi.org/10.1007/s40268-018-0254-8) contains supplementary material, which is available to authorized users.

Kyle M. Gardiner

kyle.gardiner@qut.edu.au

1 School of Pharmacy, University of Queensland, Brisbane, QLD 4102, Australia

2 Discipline of Pharmacy, Queensland University of Technology, Gardens Point Campus, Level 9, Q Block, 2 George Street, Brisbane, QLD 4000, Australia

\section{Key Points}

There exists a very high risk of bias across the individual studies considering an improvement in the gastrointestinal quality of life for patients converting between mycophenolate mofetil and enteric-coated mycophenolate sodium for maintenance immunosuppression.

This study demonstrates that there is no good evidence supporting the conversion from mycophenolate mofetil to enteric-coated mycophenolate sodium for the improvement in patient-reported gastrointestinal outcomes.

\section{Introduction}

Mycophenolate has gained widespread acceptance as the anti-proliferative immunosuppressant agent of choice in the maintenance phase of solid organ transplantation, and is increasingly being used in certain autoimmune diseases 
[1]. Advances in immunosuppressant therapies have markedly improved short-term kidney transplant outcomes, with 1-year graft survival now approaching or exceeding $95 \%$ [2-4]. Recent reports have shown, however, only minimal improvements in long-term outcomes [5]. Poor adherence to prescribed immunosuppressive regimens is regularly suggested as the reason for this [6]. A common reason for poor adherence among patients using mycophenolate is the high proportion of gastrointestinal (GI) adverse effects experienced [7]. GI-related adverse effects can lead to sub-therapeutic exposure to mycophenolate, by either dose reduction, interruption or non-adherence, placing patients at a higher risk of rejection and graft loss [8-11].

Currently, mycophenolate is available in two different salt forms: mycophenolate mofetil (MMF) and entericcoated mycophenolate sodium (EC-MPS). Following oral administration, both are hydrolysed to the active compound, mycophenolic acid (MPA). MMF was first approved for use in 1995 while EC-MPS was licensed nearly a decade later $[12,13]$. Development of EC-MPS was a response to the GI discomfort and pharmacokinetic variability classically attributed to the mofetil salt. In current practice, many clinicians will consider changing patients to EC-MPS if GI side effects are assumed related to MMF and are affecting a patient's quality of life. In the years following the initial release of mycophenolate, its utilisation rose dramatically worldwide [14]. Recently, research has shown a growing shift away from MMF towards the newer EC-MPS formulation [15]. Since introduction to market, EC-MPS has grown to account for approximately $25 \%$ of all mycophenolate dispensed in Australia [15]. It is unclear whether patients are being initiated on EC-MPS or being converted from MMF to EC-MPS. What is clear is that use of the more expensive EC-MPS is increasing over time in both absolute terms and relative to MMF [15].

To date, there have been no summaries of the available literature detailing the evidence for and against the conversion of MMF to EC-MPS for GI-related quality of life-a clinically relevant topic often discussed among transplant clinicians. The aim of this systematic review was to evaluate the risk of bias of studies comparing the GI outcomes of EC-MPS relative to MMF and to identify whether conversion from MMF to EC-MPS for reported mycophenolaterelated GI disturbances is justified based on good available evidence.

\section{Methods}

Using a pre-determined study protocol, a literature search was conducted to identify studies comparing GI-related side effects and GI-related quality of life in patients receiving
MMF and EC-MPS. The specific review questions addressed were as follows:

1. What level of bias exists for studies examining change in GI-related quality of life with conversion from MMF to EC-MPS?

2. For patients using mycophenolate, does conversion from MMF to EC-MPS improve GI-related quality of life?

An electronic search was conducted by the chief investigator (KG) using MEDLINE, Scopus, EMBASE, CINAHL and PsycINFO databases. All articles were indexed between 1 January 1995 and 30 April 2018. The search was filtered to include clinical and comparative trials involving humans. The following search terms were used for database searching: ('mycophenolic acid' [MeSH] OR 'mycophenolate' OR 'MMF' OR 'EC-MPS') AND ('gastrointestinal' OR 'GI' OR 'gastrointestinal diseases/drug therapy' [MeSH]). Bibliographic and cited reference lists were also examined for relevant sources. Duplicate entries were identified and removed before the remaining articles were screened for relevancy against the title, abstract and keywords. Full-text review, data extraction and risk of bias were completed by two independent investigators (KG and CS). Differences in extraction results and risk of bias were discussed and consensus reached. If consensus could not be reached, a third investigator (ST) decided on the outcome.

Full-text review was considered against pre-specified inclusion and exclusion criteria. Due to the scope of the review questions, the boundaries for inclusion were intentionally broad. Inclusion criteria included (a) prospective studies using either randomised or non-randomised methods and comparing MMF and EC-MPS for GI-related adverse effects or GI-related quality of life; (b) patients were receiving mycophenolate therapy for a licenced indication. Studies of both adults and children were accepted. Exclusion criteria included (a) articles published in a language other than English; (b) articles indexed as an editorial, poster abstract, case study or review; (c) articles with no primary endpoint relating to GI-related adverse effects.

Data extraction was performed using a pre-designed review form, using the following categories: first author and year of study; journal; indication for mycophenolate use; sample size; measurement tool; effect estimates at baseline and follow up (mean \pm SD for all measurement tools and associated subscale scores); equimolar dose assessment; concurrent medication; study design and methods; response rate; inclusion and exclusion criteria; funding disclosure; evidence of ethics approval and relevant comments.

Risk of bias was assessed according to The Cochrane Handbook for Systematic Reviews of Interventions [16]. This domain-based evaluation of bias considers five predominant categories of bias: selection bias, performance 
bias, detection bias, attrition bias and reporting bias. Other sources of bias considered within the boundaries of this systematic review included measurement bias and funding bias. The risk of bias for each individual study was assessed as a low risk of bias, an unclear risk of bias or a high risk of bias, according to pre-specified criteria (Supplement 1, see electronic supplementary material). Only studies considered to have a low risk of bias were included in the final synthesis of results for the purposes of answering the second research question.

Due to considerable heterogeneity in the methods applied and the specific outcomes measured across the included studies, collected data were not combined for meta-analysis. Study findings were reported according to the Preferred Reporting Items for Systematic Reviews and Meta-Analyses (PRISMA) guidelines [17].

\section{Results}

\subsection{Study Selection}

There were 3482 publications identified through database searches and two publications identified through cited reference lists, totalling 3484 records. Of these, 390 were removed due to being in a language other than English and 1315 were removed due to replication. A further 1684 records were removed as they were deemed irrelevant against the primary screening of title, abstract and keywords, leaving 95 full-text articles eligible for secondary screening against inclusion and exclusion criteria. There were 66 records that did not adhere to these pre-specified criteria. Twenty-nine studies [18-46] remained for screening of risk of bias. Of these, 26 [20-38, 40-46] studies were deemed a high or unclear risk of bias, leaving three studies considered a low risk of bias that met all necessary criteria for inclusion in the systematic review [18, 19, 39]. A flow diagram of study selection is shown in Fig. 1.

\subsection{Risk of Bias}

Overall, the risk of bias was considered high across the 29 reviewed studies. There were only three studies that were deemed a low risk of bias; however, these were not considered low risk of bias in every domain [18, 19, 39]. A summary of risk of bias is displayed in Table 1.

\subsubsection{Selection Bias}

Indiscriminate study group allocation by random sequence generation was performed in five studies $[18,19,36,39$, 44]. In each case, satisfactory allocation concealment was used $[18,19,36,39,44]$. Two further studies randomised their treatment arms; however, the method for allocation concealment was either not discussed or considered unsatisfactory $[24,40]$. One study did not provide sufficient information to make a clear decision on whether the method of randomisation or allocation concealment would reduce confounding [20]. The remaining 21 studies allocated patients in a non-randomised manner [21-23, $25-35,37,38,41-43,45,46]$. The enrolled population was selected using well defined clinical criteria in 15 studies [18, 19, 22, 24, 30, 33-37, 40, 43-46]. In four studies, neither the method used to enrol participants nor the selection criteria were explicitly defined $[21,32,38$, 39]. There were ten studies that enrolled their population using methods that did not account for bias introduced from an intentionally skewed proportion of diseased individuals [20, 23, 25-29, 31, 41, 42]. The vast majority of studies limited sampling bias by clearly specifying the study population and by ensuring the sample was representative of the study population $[18-20,22-25,27$, $28,30,32-46]$. In contrast, the vast majority introduced sampling bias by not conducting sample size analysis [20, $21,23,24,26-35,37,38,40,42-46]$. Of the seven studies that did perform sample size analysis, only six recruited the required sample to meet power calculations $[18,19$, $22,25,39,41]$. Overall, there was a high risk of selection bias across the 29 studies.

\subsubsection{Performance and Detection Bias}

Three studies were free from performance and detection bias as they involved both double blinding and double dummy control $[18,19,39]$. This ensured that all participants, outcome assessors and healthcare providers were blinded to the intervention allocations. A further two studies reported successful blinding of their participants; however, as the two forms of mycophenolate appear quite different, it is unclear as to whether a lack of a dummy control affected study performance and detection bias [24, 44]. The remaining 24 studies did not blind their participants, outcomes assessors or healthcare providers [20-23, 25-43, 45, 46]. Overall, there was a high risk of performance and detection bias across the 29 studies analysed.

\subsubsection{Attrition Bias}

Loss to follow up and withdrawal rates were generally quite low with 20 studies reporting a withdrawal rate of $15 \%$ or less [18, 19, 24, 25, 27-30, 32, 35-37, 39-45]. In each case, the underlying reason for attrition was adequately described and appropriate methods were employed to reduce bias. In seven cases, no withdrawal rate was provided nor was there sufficient information given to assess the influence of 


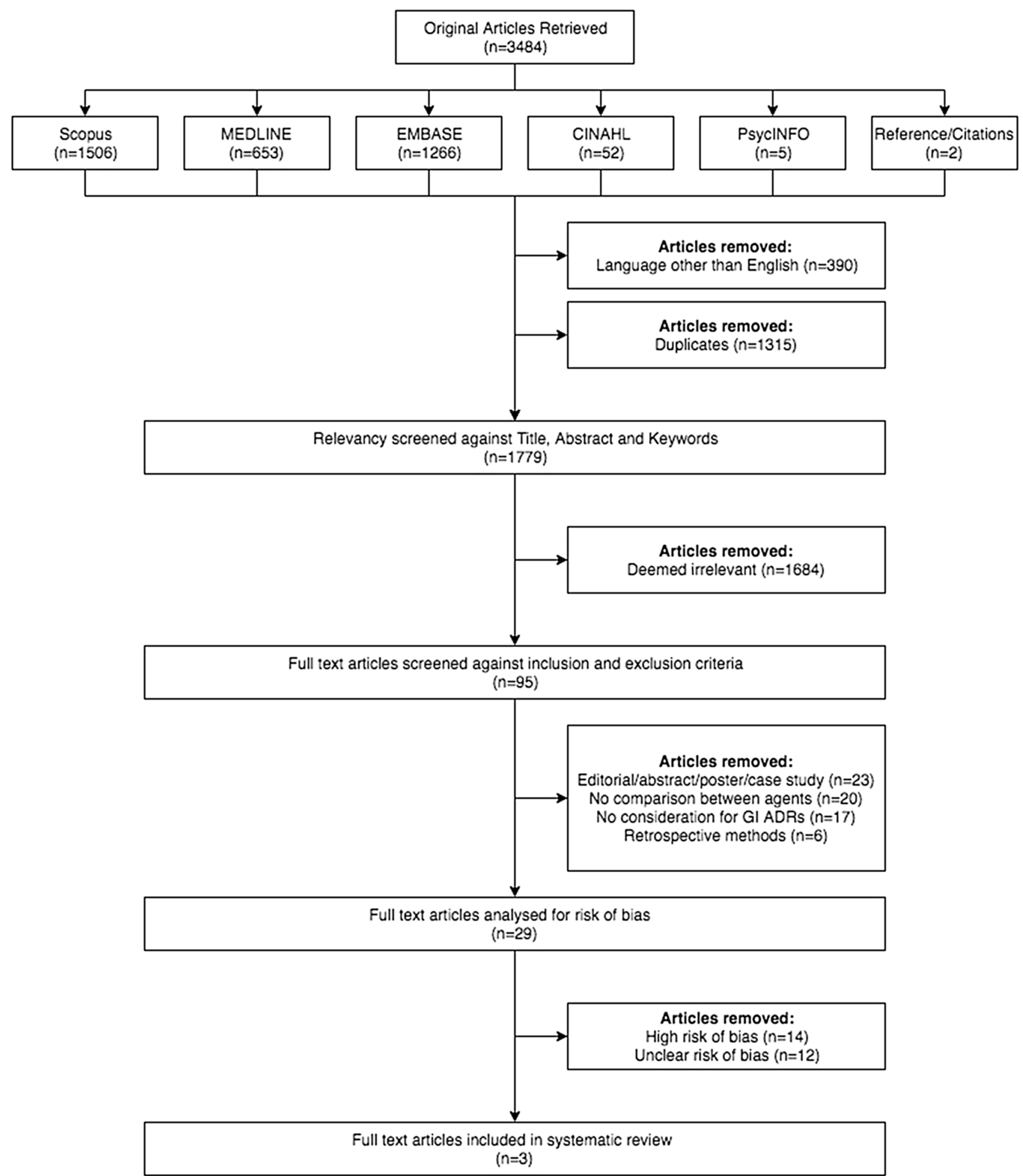

Fig. 1 PRISMA flow diagram of included studies

attrition on bias [20, 21, 23, 26, 31, 33, 38]. Three studies reported conclusions that did not adequately reflect the level of, or give reasons for attrition [22, 34, 46]. Overall, there was a low risk of attrition bias.

\subsubsection{Reporting Bias}

Selective reporting was apparent in 14 studies [22, 26, 30, $31,33-35,37-41,45,46]$. In the majority of cases, primary 
Table 1 A summary of risk of study bias for individual studies where red is a high risk of bias, yellow is an unclear risk of bias and green is a low risk of bias

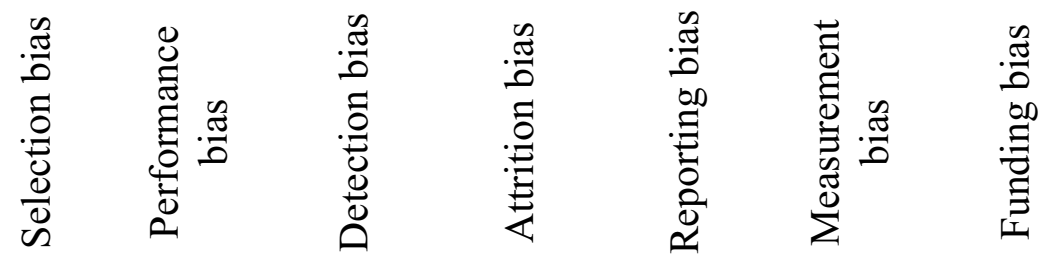

\begin{tabular}{|c|c|c|c|c|c|c|c|}
\hline Budde, 2004[18] & + & + & + & + & + & (2) & - \\
\hline Salvadori, 2004[19] & + & + & + & + & + & C) & - \\
\hline Kamar, 2005[20] & ? & 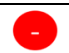 & O & (2) & $(2)$ & O & O \\
\hline Calvo, 2006[21] & C & - & $\odot$ & (2) & (2) & O & (2) \\
\hline Chan, 2006[22] & (3) & - & - & - & - & + & - \\
\hline Dumortier, 2006[23] & O & 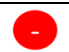 & O & (2) & + & O & 8 \\
\hline Kobashigawa, 2006[24] & (2) & (2) & O & + & + & O & - \\
\hline Bolin, 2007[25] & (2) & e & e & + & + & (?) & e \\
\hline Cofan, 2007[26] & $\Theta$ & $\Theta$ & O & $(2)$ & - & $(3)$ & - \\
\hline Darji, 2008[27] & e & e & e & + & (2) & (2) & (2) \\
\hline Pape, 2008[28] & - & C) & C & + & + & (2) & (2) \\
\hline $\begin{array}{l}\text { Barrera-Pulido, } \\
\text { 2009[29] }\end{array}$ & C & - & O & + & (2) & O & (3) \\
\hline Bilodeau, 2009[30] & C & C & O & + & C & (2) & - \\
\hline Burg, 2009[31] & C & - & O & $(3)$ & - & O & - \\
\hline Meneses, 2009[32] & C & $\odot$ & 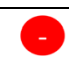 & + & + & - & $(3)$ \\
\hline Doria, 2009[33] & C & C- & 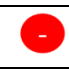 & (1) & - & (2) & $\odot$ \\
\hline Robaeys, 2009[34] & C & C) & O & C & O & O & $(3)$ \\
\hline Sabbatini, 2009[35] & - & - & - & + & - & (3) & (2) \\
\hline Shehata, 2009[36] & + & O & $\Theta$ & + & + & (2) & O \\
\hline Hwang, 2010[37] & O & C & - & + & $\theta$ & (2) & $\Theta$ \\
\hline Reyes, 2010[38] & C & C & O & $(3)$ & - & (8) & (3) \\
\hline Langone, 2011[39] & + & + & + & + & C & + & $\Theta$ \\
\hline Ortega, 2011[40] & $(2)$ & C & O & + & O & $(3)$ & 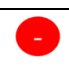 \\
\hline Reinke, 2011[41] & (?) & $\odot$ & - & + & - & $(2)$ & $(3)$ \\
\hline Toledo, 2012[42] & C & O & O & + & + & 8 & - \\
\hline Bunnapradist, 2014[43] & O & C & O & + & + & $(3)$ & - \\
\hline Lopez-Solis, 2014[44] & + & (3) & $(3)$ & + & (3) & $(3)$ & $\odot$ \\
\hline Sterneck, 2014[45] & O & 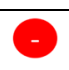 & $\Theta$ & + & - & 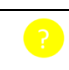 & O \\
\hline Manger, 2015[46] & O & O & 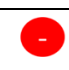 & O & - & $(3)$ & $\Theta$ \\
\hline
\end{tabular}


outcomes and associated statistics were only partially reported. Another five studies had some degree of selective reporting, but the influence on conclusions were unclear [20, $21,27,29,44]$. Ten studies reported all primary and secondary outcomes with reported conclusions reflecting the results obtained [18, 19, 23-25, 28, 32, 36, 42, 43]. Overall, there was a high risk of reporting bias across the 29 studies analysed.

\subsubsection{Other Biases}

When considering measurement bias, 20 studies used a validated tool to measure the change in GI symptoms between baseline and follow-up [22, 25-28, 30, 33-46]. However, only two of these studies utilised methods that adequately limited additional recall bias, a potential limitation of using patient-reported outcome measures in an unblinded manner $[22,39]$. As such, it is unclear to what extent measurement bias influenced conclusions. Finally, 19 studies declared funding by the manufacturer of the agent being investigated [18-20, 22, 24-26, 30, 31, 33, 36, 37, 39, 40, 42-46]. The remaining ten studies provided no written declaration of funding [21, 23, 27-29, 32, 34, 35, 38, 41]. Overall, there exists a high risk of funding bias across the 29 studies analysed.

\subsection{Effect of Intervention}

Overall, 1141 individuals participated in three trials included in this systematic review. The average age of participants in these trials ranged between 46 and 49 years. The proportion of males and females was $60 \%$ and $40 \%$, respectively. All three studies compared the use of MMF and EC-MPS following renal transplant. In each case, an equimolar dose of mycophenolate was used. Budde et al. [18] reported a mean time since transplantation of 863.9 days (SD 830.9) and 843.9 days (SD 764.6) for MMF and EC-MPS, respectively. Langone et al. [39] reported a mean time since transplantation of 1011 days (SD 1176) and 1136.2 days (SD 1269.2) for MMF and EC-MPS, respectively. In contrast, Salvadori et al. [19] recruited participants prior to their primary transplant. There were 123 total discontinuations across the three studies, with individual attrition ranging from 6 to $15 \%$. In all studies, patients concurrently received a calcineurin inhibitor (cyclosporin or tacrolimus) with or without corticosteroids. As the Salvadori et al. [19] study coincided with induction immunosuppression, these patients were also permitted to receive antithymocyte and antilymphocyte induction immunosuppressive agents. All three trials involved randomised enrolment of participants in a 1:1 ratio into two treatment arms: group 1 (control) - participants to receive MMF + matching ECMPS placebo + calcineurin inhibitor \pm corticosteroids; group 2 (intervention) - participants to receive EC-MPS + matching
MMF placebo + calcineurin inhibitor \pm corticosteroids. The major primary efficacy and safety endpoints of the three included studies are now discussed. These include GI quality of life, graft failure, infection, malignancy and haematological abnormalities. Study characteristics and results are summarised in Table 2.

\subsubsection{Gastrointestinal Quality of Life}

All three included studies considered the impact of EC-MPS and MMF on GI quality of life [18, 19, 39]. More specifically, each of the three studies considered outcomes at different time points along the post-randomisation continuum (Fig. 2). Langone et al. [39] reported that at 30 days post-conversion, the proportion of any GI-related adverse effects was $39 \%$ and $46 \%$ for the EC-MPS and MMF groups, respectively. Budde et al. [18] reported that (a) at 3 months post-conversion, the proportion of any GI-related adverse effect was $26.4 \%$ and $20.9 \%$ for the EC-MPS and MMF groups, respectively; (b) at 6 months post-conversion, the proportion of any GI-related adverse effect was $28.9 \%$ and $27.6 \%$ for the EC-MPS and MMF groups, respectively; and (c) at 12 months post-conversion, the proportion of any GI-related adverse effect was $60.4 \%$ and $61.3 \%$ for the EC-MPS and MMF groups, respectively. Salvadori et al. [19] reported that at 12 months post-randomisation, the proportion of any GI-related adverse effect was $80.8 \%$ and $80.0 \%$ for the EC-MPS and MMF groups, respectively. In each case, the differences observed were not statistically significant.

Langone et al. [39] utilised two validated patient-reported outcome measures [the Gastrointestinal Symptom Rating Scale (GSRS) and the Gastrointestinal Quality of Life Index (GIQLI)] to access the difference in GI-related quality of life between baseline and 30 days post-randomisation. Both control and intervention groups experienced a statistically significant decrease in GSRS between baseline and day $30(p<0.001)$; yet, the between-group differences were not considered statistically significant. Of the five GSRS subdomains, EC-MPS usage was associated with a significantly greater improvement in indigestion syndromes relative to MMF (mean change: $\mathrm{EC}-$ MPS $0.7 \pm 1.2$, MMF $0.5 \pm 1.4 ; p=0.02$ ). There was no significant differences in diarrhoea, constipation, abdominal pain and reflux syndromes. Similarly, both control and intervention groups displayed statistically significant improvements in the GIQLI ( $p=0.003$ ); however, between-group differences were again not significant.

\section{Discussion}

\subsection{Summary of Main Results}

In this systematic review, the utility of using EC-MPS to replace MMF as maintenance immunosuppression for the 


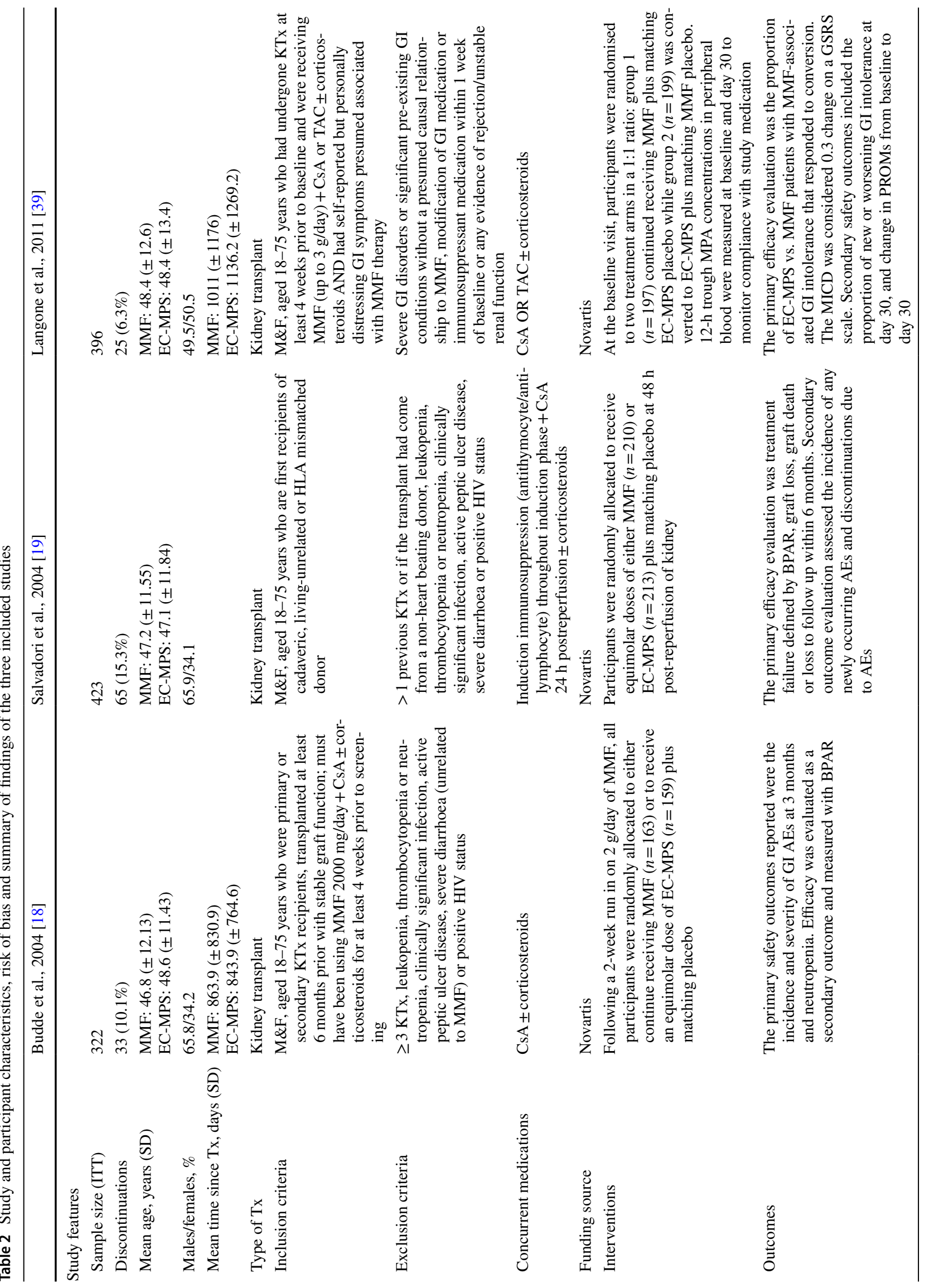




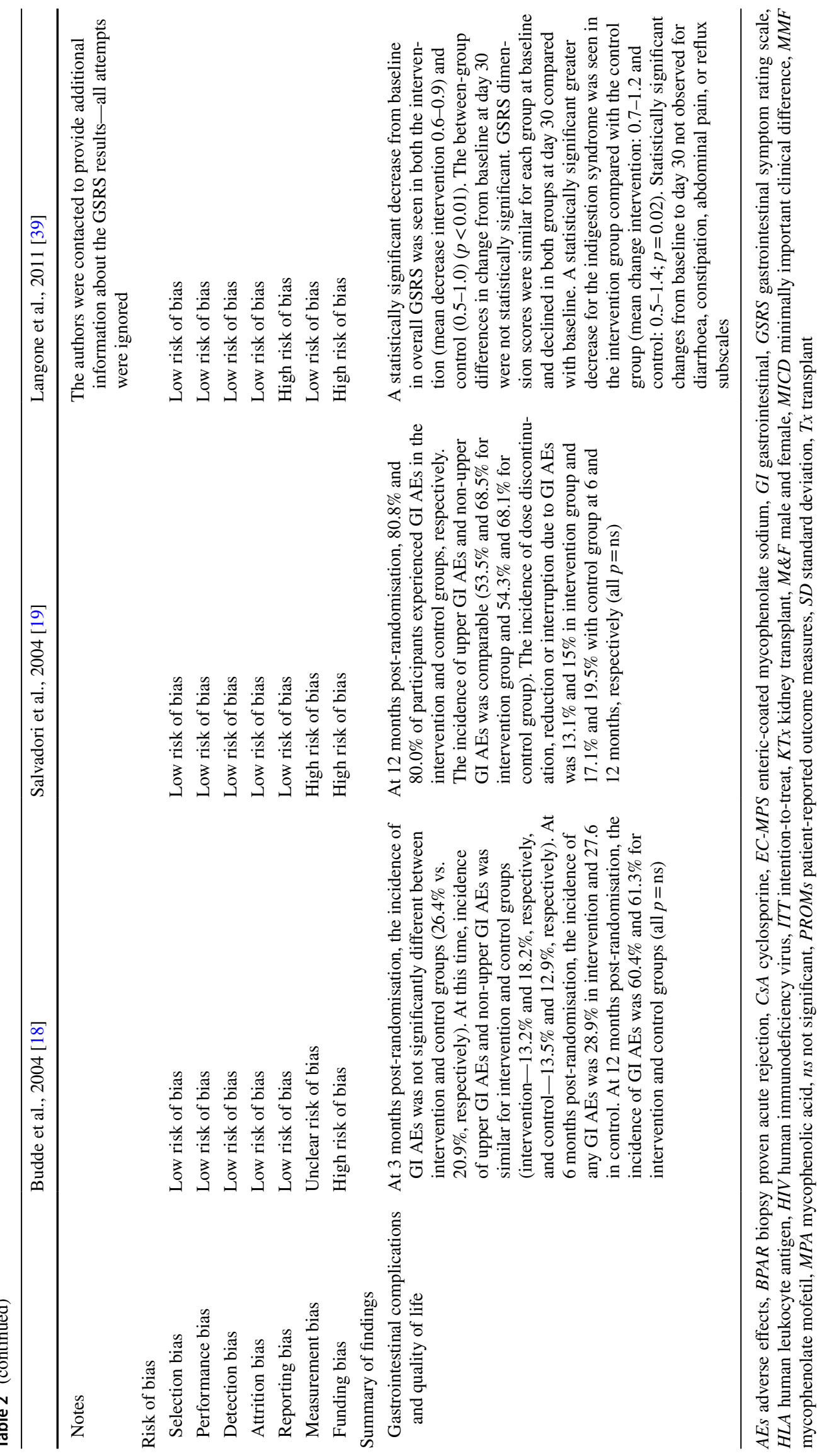


Fig. 2 A comparison of the proportion of any recorded GI-related adverse effect for MMF and EC-MPS. Bar A corresponds with the results from Langone et al. [39]; bars $\mathrm{B}, \mathrm{C}$ and $\mathrm{D}$ correspond with the results from Budde et al. [18]; bar E corresponds with results from Salvadori et al. [19]. Direct comparisons for each analogous pair were not significant in all cases. EC-MPS enteric-coated mycophenolate sodium, GI gastrointestinal, $M M F$ mycophenolate mofetil

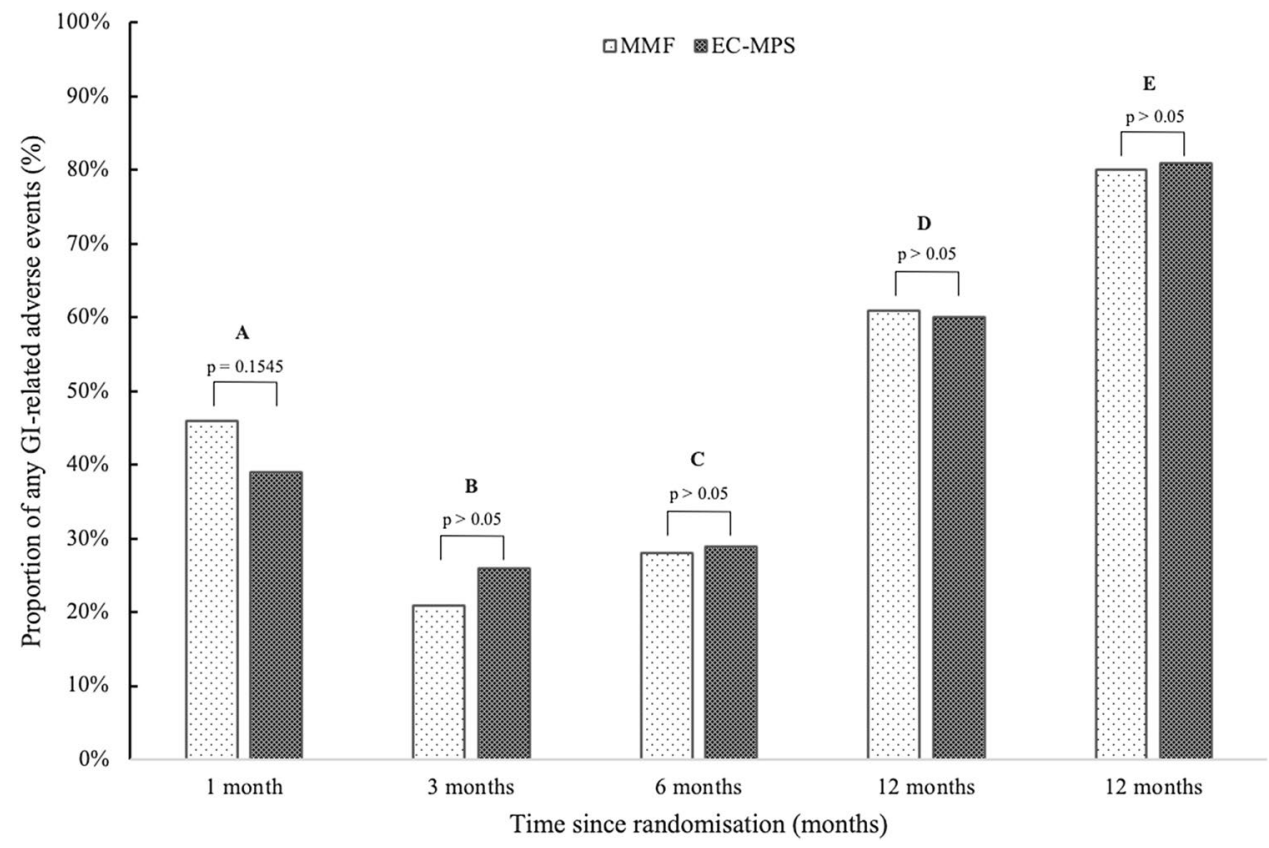

purposes of reducing GI-related adverse effects was examined. The review initially considered the risk of bias for all studies comparing MMF and EC-MPS for GI tolerability. The review then consolidated the results of studies with a low risk of bias to compare the GI tolerability in patients converted from MMF to EC-MPS and those initiated on either MMF or EC-MPS. Primarily, the review found an overall high risk of bias across the 29 studies comparing MMF and EC-MPS for GI tolerability. When considering the five domains of the Cochrane domain-based evaluation of bias, only attrition bias was thought to be robust across the 29 studies. The other four domains were considered to have a high likelihood of biasing the results and conclusions made. Furthermore, the extent of funding bias would carry a high risk of bias while there was an overall unclear influence of measurement bias on conclusions made. The studies, and thus the results and conclusions regarding the conversion from MMF to EC-MPS for the improvement in GI-related quality of life are heavily influenced by bias making the applicability of individual studies limited.

The second main finding, from aggregated data in the studies with low risk of bias, was no significant difference in the proportion of GI-related adverse effects and no improvement in overall GI-related quality of life in patients converted from MMF to EC-MPS or those initiated on either agent. As expected, the duration of exposure to either MMF or EC-MPS led to an increase in the probability of a patient experiencing a GI-related adverse effect; however, this increase was consistent between both intervention and control groups. Furthermore, irrespective of the patient-reported outcome measure used, both intervention and control groups saw a significant improvement in overall GI-related quality of life, whereas between-group differences remained insignificant. These consolidated results suggest that the parallel comparison of equimolar MMF and EC-MPS leads to similar GI-related tolerability.

\subsection{Agreements and Disagreements with Other Studies and Reviews}

This is the first systematic review comparing the GI-related quality of life for patients receiving mycophenolate. Findings from this systematic review differ from conclusions reported in the majority of individual studies. The vast majority of these individual studies were deemed as having either a high risk of bias or an unclear risk of bias and reported results favouring the conversion to EC-MPS, stating it as superior for GI-related quality of life relative to MMF. For example, Burg et al. [31] concluded that a considerable number of patients suffered GI complications during MMF therapy; however, most of these patients reported improvement or total disappearance of these adverse effects post-conversion to EC-MPS. Chan et al. [22] concluded that following conversion to EC-MPS, the overall GSRS and GIQLI scores as well as all GSRS and GIQLI subscale scores improved significantly between baseline and follow-up. The GI quality of life for participants maintained on MMF remained unchanged over the same time course. Cofan et al. [26] reported a significant improvement in the overall GIQLI following conversion from MMF to EC-MPS. All GIQLI subscales indicated significant improvements except the social function subscale. In contrast, the studies deemed as having 
a low risk of bias or those that were borderline between an unclear and low risk of bias generally concluded no difference between intervention and control groups. In each example above, the study presents conclusions that contradict the results of this systematic review, yet, in each example, the study was considered to have a high risk of bias. The overall high risk of bias presented by this review should limit the applicability of individual studies.

\subsection{Overall Completeness and Applicability of Results}

The strength of this review was the methods applied. A pre-specified study protocol was used to identify all studies comparing the GI-related quality of life for MMF and ECMPS. Full-text review, data extraction and risk of bias analysis was completed by two independent authors. Although inclusion and exclusion criteria were intentionally broad, this allowed the review to capture all literature considering the conversion from MMF to EC-MPS for the improvement of GI-related quality of life. A thorough review process allowed only studies with a low risk of bias to be included in the systematic review. Due to the overall high risk of bias observed, only three studies could be included in the actual systematic review. Although these studies were methodologically sound, substantial heterogeneity in the results obtained limited the capacity for pooling of results for meta-analysis.

The concurrent use of corticosteroids and one of either cyclosporin or tacrolimus with MPA is commonplace during the maintenance phase of immunosuppression, yet these agents are all known to influence the prevalence of GI-related adverse events. Additionally, cyclosporin and tacrolimus can both influence MPA trough concentrations, albeit to differing extents. As such, these agents each have the capacity to confound the direct comparisons between MMF and EC-MPS. Budde et al. [18] and Salvadori et al. [19] demonstrated no significant inter-group difference between corticosteroid and cyclosporin doses, nor was there a significant difference in cyclosporin trough concentration. Langone et al. [39] permitted the use of either cyclosporin or tacrolimus; however, sensitivity analyses demonstrated no effect upon results. Overall, it is unlikely that the comparison of MMF and EC-MPS for GI-related adverse events has been confounded by either calcineurin inhibitor or corticosteroid co-administration.

The three included studies all utilised mycophenolate as a maintenance immunosuppressant post-renal transplant where equimolar doses of MMF and EC-MPS were used. In most cases, patients with concurrent bacterial infection, positive human immunodeficiency virus status and malignancy were excluded. Additionally, the average age of all three samples largely excluded children and the elderly. Therefore, the results of this review are only explicitly applicable to adult patients whom received a renal transplant, are otherwise well and are using mycophenolate as maintenance immunosuppression.

\subsection{Implications for Practice}

On the basis of the three rigorous studies included in this systematic review, the incidence and severity of GI-related adverse effects appears to be similar between MMF and EC-MPS usage. Furthermore, the risk of graft failure, infection, malignancy and haematological abnormalities remains similar between the two salt forms of mycophenolate. Notwithstanding this, GI complications are a common issue in patients being treated with mycophenolate, and this can lead to a reduction in adherence [7]. Primarily, these results may offer some additional insight into the growing commentary surrounding MPA trough and plasma monitoring for the purposes of improving GI-related quality of life and subsequent adherence. Yet, given the intrinsic link between reduced adherence and graft failure, this is an issue that needs immediate clinical attention [8-10]. Patients experiencing GI-related adverse effects with mycophenolate usage should primarily be encouraged to take this medicine with food. To reduce unnecessary pharmacokinetic variability, consistency should be established between the fed and fasted states of administration. Failing this, the patient should be encouraged to switch from twice daily administration to three or four divided doses. Only if these two options fail should the clinician consider changing between salt forms of mycophenolate. Although this systematic review suggests the incidence and severity of GI-related complications is similar, the risks associated with conversion are minimal. Additionally, given the unblinded nature of clinical practice, there might be a proportion of patients who would benefit from this conversion. Additionally, despite the prevailing bias, a number of studies highlighted a trend towards the use of EC-MPS allowing higher total daily doses to be achieved [30, 35, 40]. The rationale is that if a patient's mean daily dose of mycophenolate can increase, so can the individual's longterm allograft outcomes. Although this theory is anchored by sound physiology, it operates under the assumption that EC-MPS has a greater GI tolerability profile than MMF. This systematic review has shown that the incidence of GI-related complications is similar between MMF and ECMPS. GI complications are common with mycophenolate and are the principle cause of non-adherence. Introducing higher EC-MPS doses will likely be of no benefit if patients remain non-adherent to the prescribed regimen. Nevertheless, dose adjustments should be made on a caseby-case basis according to the patient's tolerability. 


\section{Conclusion}

This systematic review examined the evidence around conversion from MMF to EC-MPS and the issue of GI-related quality of life. It sought to answer two questions.

1. What level of bias exists for studies examining change in GI-related quality of life with conversion from MMF to EC-MPS?

Overall, there exists a high risk of bias across the studies reviewed in this paper. This level of bias makes it difficult for clinicians to have confidence in the results of individual studies. This lack of confidence is reinforced by the conflicting nature of results from studies with a low risk of bias verses those with a high risk of bias. This review has compiled the studies considered a low risk of bias to provide robust recommendations for the conversion of MMF to EC-MPS.

2. For patients using mycophenolate, does conversion from MMF to EC-MPS improve GI-related quality of life?

The results of this systematic review demonstrate that there is no difference between GI-related quality of life for patients using MMF and EC-MPS as maintenance immunosuppression. This includes patients who have been converted from MMF to EC-MPS as well as patients who have been initiated on either agent. If GI-related complications occur in a patient on MMF, other avenues should be explored first before conversion takes place. The proportion of patients who will benefit from conversion is likely to be minimal. These data are limited to renal transplant recipients; extrapolation to patients using mycophenolate for other solid organ transplants or lupus nephritis requires more research.

Author contributions KMG participated in study design, performed research/study, collected data, analysed data and wrote the manuscript; SET participated in the study design, performed research/study and revised the manuscript; CES participated in study design, performed research/study, analysed data and revised the manuscript.

Funding No direct funding was received for this study. KMG received a salary as a practising pharmacist and an Australian postgraduate award scholarship for $\mathrm{PhD}$ studies at the Queensland University of Technology, SET and CES received salaries from the University of Queensland.

\section{Compliance with Ethical Standards}

Conflict of interest Kyle Gardiner, Susan Tett and Christine Staatz declare that they have no conflict of interest.
Open Access This article is distributed under the terms of the Creative Commons Attribution-NonCommercial 4.0 International License (http://creativecommons.org/licenses/by-nc/4.0/), which permits any noncommercial use, distribution, and reproduction in any medium, provided you give appropriate credit to the original author(s) and the source, provide a link to the Creative Commons license, and indicate if changes were made.

\section{References}

1. Wagner M, Earley AK, Webster AC, et al. Mycophenolic acid versus azathioprine as primary immunosuppression for kidney transplant recipients. Cochrane Database Syst Rev. 2015;12:1361-6137.

2. Australia and New Zealand Dialysis and Transplant Registry. Thirty Eighth Annual Report: Chapter 8. Transplantation. 2016;38:1-21.

3. Caskey F, Castledine C, Dawnay A, et al. 18th Annual Report of the Renal Association. Nephron. 2016;132:115-31.

4. Saran R, Li Y, Robinson B. US Renal Data System 2015 Annual Data Report: Epidemiology of Kidney Disease in the United States. Am J Kidney Dis. 2016;67(3 suppl 1):S1-305.

5. Meier-Kriesche HU, Schold JD, Srinivas TR, et al. Lack of improvement in renal allograft survival despite a marked decrease in acute rejection rates over the most recent era. Am J Transpl. 2004;4(3):378-83.

6. Sellares J, de Freitas DG, Mengel M, et al. Understanding the causes of kidney transplant failure: the dominant role of antibody-mediated rejection and nonadherence. Am J Transpl. 2012;12(2):388-99.

7. Behrend M. Adverse gastrointestinal effects of mycophenolate mofetil: aetiology, incidence and management. Drug Saf. 2001;24(9):645-63.

8. Pelletier RP, Akin B, Henry ML, et al. The impact of mycophenolate mofetil dosing patterns on clinical outcome after renal transplantation. Clin Transpl. 2003;17(3):200-5.

9. Tierce JC, Porterfield-Baxa J, Petrilla AA, et al. Impact of mycophenolate mofetil (MMF)-related gastrointestinal complications and MMF dose alterations on transplant outcomes and healthcare costs in renal transplant recipients. Clin Transpl. 2005;19(6):779-84.

10. Machnicki G, Ricci JF, Brennan DC, et al. Economic impact and long-term graft outcomes of mycophenolate mofetil dosage modifications following gastrointestinal complications in renal transplant recipients. Pharmacoeconomics. 2008;26(11):951-67.

11. Rivera F, Anaya S. Lupus nephritis flare in young patients: relapse or nonadherence to treatment? Int J Nephrol Renovasc Dis. 2014;7:117-21.

12. Roche Pharmaceuticals. CellCept ${ }^{\circledR}$ Product Information; 2012. http://www.roche-australia.com/content/dam/internet/corporate/ roche/en_AU/files/transplant/cellcept-pi.pdf. Accessed 25 Nov 2017.

13. Novartis Australia. Myfortic ${ }^{\circledR}$ Product Information; 2012. http:// www.tga.gov.au/pdf/auspar/auspar-mycophenolate-sodium-13051 7-pi.pdf. Accessed 25 Nov 2017.

14. U.S. Department of Health and Human Services. OPTN/ SRTR 2015 Annual Data Report. Kidney Am J Transpl. 2017;17(S1):21-116.

15. Gardiner KM, Tett SE, Staatz CE. Multinational evaluation of mycophenolic acid, tacrolimus, cyclosporin, sirolimus, and everolimus utilization. Ann Transpl. 2016;21:1-11. 
16. Higgins JP, Altman DG, Gotzsche PC, et al. The Cochrane Collaboration's tool for assessing risk of bias in randomised trials. BMJ. 2011;343:d5928.

17. Moher D, Liberati A, Tetzlaff J, et al. Preferred reporting items for systematic reviews and meta-analyses: the PRISMA statement. Ann Intern Med. 2009;151(4):264-269, w264.

18. Budde K, Curtis J, Knoll G, et al. Enteric-coated mycophenolate sodium can be safely administered in maintenance renal transplant patients: results of a 1-year study. Am J Transpl. 2004;4(2):237-43

19. Salvadori M, Holzer H, de Mattos A, et al. Enteric-coated mycophenolate sodium is therapeutically equivalent to mycophenolate mofetil in de novo renal transplant patients. Am J Transpl. 2004;4(2):231-6.

20. Kamar N, Oufroukhi L, Faure P, et al. Questionnaire-based evaluation of gastrointestinal disorders in de novo renaltransplant patients receiving either mycophenolate mofetil or enteric-coated mycophenolate sodium. Nephrol Dial Transpl. 2005;20(10):2231-6.

21. Calvo N, Sanchez-Fructuoso AI, Conesa J, et al. Renal transplant patients with gastrointestinal intolerability to mycophenolate mofetil: conversion to enteric-coated mycophenolate sodium. Transpl Proc. 2006;38(8):2396-7.

22. Chan L, Mulgaonkar S, Walker R, et al. Patient-reported gastrointestinal symptom burden and health-related quality of life following conversion from mycophenolate mofetil to enteric-coated mycophenolate sodium. Transplantation. 2006;81(9):1290-7.

23. Dumortier J, Gagnieu MC, Salandre J, et al. Conversion from mycophenolate mofetil to enteric-coated mycophenolate sodium in liver transplant patients presenting gastrointestinal disorders: a pilot study. Liver Transpl. 2006;12(9):1342-6.

24. Kobashigawa JA, Renlund DG, Gerosa G, et al. Similar efficacy and safety of enteric-coated mycophenolate sodium (EC-MPS, Myfortic) compared with mycophenolate mofetil (MMF) in de novo heart transplant recipients: results of a 12-month, singleblind, randomized, parallel-group, multicenter study. J Heart Lung Transpl. 2006;25(8):935-41.

25. Bolin P, Tanriover B, Zibari GB, et al. Improvement in 3-month patient-reported gastrointestinal symptoms after conversion from mycophenolate mofetil to enteric-coated mycophenolate sodium in renal transplant patients. Transplantation. 2007;84(11):1443-51.

26. Cofan F, Rosich E, Arias M, et al. Quality of life in renal transplant recipients following conversion from mycophenolate mofetil to enteric-coated mycophenolate sodium. Transpl Proc. 2007;39(7):2179-81.

27. Darji P, Vijayaraghavan R, Thiagarajan CM, et al. Conversion from mycophenolate mofetil to enteric-coated mycophenolate sodium in renal transplant recipients with gastrointestinal tract disorders. Transpl Proc. 2008;40(7):2262-7.

28. Pape L, Ahlenstiel T, Kreuzer M, et al. Improved gastrointestinal symptom burden after conversion from mycophenolate mofetil to enteric-coated mycophenolate sodium in kidney transplanted children. Pediatr Transpl. 2008;12(6):640-2.

29. Barrera-Pulido L, Alamo-Martínez JM, Marín-Gómez LM, et al. Switching from mycophenolate mofetil to enteric-coated mycophenolate sodium in liver transplant patients with gastrointestinal complications. Transpl Proc. 2009;41(6):2192-4.

30. Bilodeau JF, Montambault P, Wolff JL, et al. Evaluation of tolerability and ability to increase immunosuppression in renal transplant patients converted from mycophenolate mofetil to entericcoated mycophenolate sodium. Transpl Proc. 2009;41(9):3683-9.

31. Burg M, Säemann MD, Wieser C, et al. Enteric-coated mycophenolate sodium reduces gastrointestinal symptoms in renal transplant patients. Transpl Proc. 2009;41(10):4159-64.

32. De Paula Meneses R, Halusch Kotsifas C. Benefits of conversion from mycophenolate mofetil to enteric-coated mycophenolate sodium in pediatric renal transplant patients with stable graft function. Pediatr Transpl. 2009;13(2):188-93.

33. Doria C, Ramirez CB, Frank AM, et al. Use of enteric-coated mycophenolate sodium in liver transplant patients with intestinal intolerance caused by mycophenolate mofetil. Clin Transpl. 2009;23(6):882-6.

34. Robaeys G, Cassiman D, Verslype C, et al. Successful conversion from mycophenolate mofetil to enteric-coated mycophenolate sodium (myfortic) in liver transplant patients with gastrointestinal side effects. Transpl Proc. 2009;41(2):610-3.

35. Sabbatini M, Capone D, Gallo R, et al. EC-MPS permits lower gastrointestinal symptom burden despite higher MPA exposure in patients with severe MMF-related gastrointestinal side-effects. Fundam Clin Pharmacol. 2009;23(5):617-24.

36. Shehata M, Bhandari S, Venkat-Raman G, et al. Effect of conversion from mycophenolate mofetil to enteric-coated mycophenolate sodium on maximum tolerated dose and gastrointestinal symptoms following kidney transplantation. Transpl Int. 2009;22(8):821-30.

37. Hwang HS, Hyoung BJ, Kim S, et al. Improved gastrointestinal symptoms and quality of life after conversion from mycophenolate mofetil to enteric-coated mycophenolate sodium in renal transplant patients receiving tacrolimus. J Korean Med Sci. 2010;25(12):1759-65.

38. Reyes H, Hernández AM, Valverde S, et al. Efficacy and safety of conversion of mycophenolate mofetil to enteric-coated mycophenolate sodium in Mexican renal transplant children. Pediatr Transpl. 2010;14(6):746-52.

39. Langone AJ, Chan L, Bolin P, et al. Enteric-coated mycophenolate sodium versus mycophenolate mofetil in renal transplant recipients experiencing gastrointestinal intolerance: a multicenter, double-blind, randomized study. Transplantation. 2011;91(4):470-8.

40. Ortega F, Sánchez-Fructuoso A, Cruzado JM, et al. Gastrointestinal quality of life improvement of renal transplant recipients converted from mycophenolate mofetil to enteric-coated mycophenolate sodium drugs or agents: mycophenolate mofetil and enteric-coated mycophenolate sodium. Transplantation. 2011;92(4):426-32.

41. Reinke P, Budde K, Hugo C, et al. Reduction of gastrointestinal complications in renal graft recipients after conversion from mycophenolate mofetil to enteric-coated mycophenolate sodium. Transpl Proc. 2011;43(5):1641-6.

42. Toledo AH, Hendrix L, Buchholz V, et al. Improvement of gastrointestinal symptoms after conversion from mycophenolate mofetil to enteric-coated mycophenolate sodium in liver transplant patients. Clin Transpl. 2012;26(1):156-63.

43. Bunnapradist S, Sampaio MS, Wilkinson AH, et al. Changes in the small bowel of symptomatic kidney transplant recipients converted from mycophenolate mofetil to enteric-coated mycophenolate sodium. Am J Nephrol. 2014;40(2):184-90.

44. Lopez-Solis R, DeVera M, Steel J, et al. Gastrointestinal side effects in liver transplant recipients taking enteric-coated mycophenolate sodium vs. mycophenolate mofetil. Clin Transpl. 2014;28(7):783-8.

45. Sterneck M, Settmacher U, Ganten T, et al. Improvement in gastrointestinal and health-related quality of life outcomes after conversion from mycophenolate mofetil to enteric-coated mycophenolate sodium in liver transplant recipients. Transpl Proc. 2014;46(1):234-40.

46. Manger B, Hiepe F, Schneider M, et al. Impact of switching from mycophenolate mofetil to enteric-coated mycophenolate sodium on gastrointestinal side effects in patients with autoimmune disease: a phase III, open-label, single-arm, multicenter study. Clin Exp Gastroenterol. 2015;8:205-13. 